\title{
Pratiques paysannes de gestion des cultivars de manioc (Manihot esculenta Crantz) au Sud du Tchad
}

\author{
Djirabaye NADJIAM ${ }^{1,2^{*}}$, Mariama DIALLO ${ }^{3}$, Jean Marie Mbaïlao MBAÏGUINAM ${ }^{4}$ et \\ Aliou GUISSE ${ }^{2,5}$ \\ ${ }^{1}$ Institut Tchadien de Recherche Agronomique pour le Développement (ITRAD), BP: 5400, N'Djaména, Tchad. \\ ${ }^{2}$ Département de Biologie Végétale, Faculté des Sciences et Techniques (FST), Université Cheikh Anta Diop \\ (UCAD), BP: 5005 Dakar-Fann, Sénégal. \\ ${ }^{3}$ Section Productions Végétales et Agronomie, UFR S2ATA Université Gaston Berger de Saint Louis, Sénégal. \\ ${ }^{4}$ Université des Sciences et des Technologies, Bp: 20 Ati, Batha, Tchad. \\ ${ }^{5}$ Observatoire Homme Milieux Téssekeré (OHM-Téssekeré), Université Cheikh Anta Diop de Dakar, B.P. 5005 \\ Dakar-Fann, Sénégal. \\ *Auteur correspondant, E-mail : djibsna27@gmail.com ; djirabaye.nadjiam@ucad.edu.sn, \\ Tel. : +23566291728.
}

\section{REMERCIEMENTS}

Nous adressons nos sincères remerciements aux responsables de l'ITRAD, au PRASAC et à l'UE pour la bourse qui nous a été accordée pour ce travail.

\section{RESUME}

Dans les petites exploitations familiales, les paysans détiennent un nombre important de cultivars de manioc inexploités par la recherche. L'objectif de cette étude est d'évaluer le niveau de la diversité du manioc et d'en analyser les pratiques locales de gestion. Une enquête suivant une approche participative avec des questionnaires a été conduite auprès de 636 paysans dans 43 villages. Une importante diversité variétale a été révélée par l'indice de Shannon (3,38). Soixante-quinze noms vernaculaires ont été inventoriés. Des différences hautement significatives ont été observées pour le nombre de cultivars qui varie de 2 à 12 par village (6 en moyenne). La variation entre ménages est faible, 1 à 4 cultivars ( 2 en moyenne). Le nombre de cultivars disparus varie de 1 à 14 (3,81 en moyenne) par village. La perte de la diversité est en moyenne de 18,20\% et varie entre $11 \%$ et $50 \%$ selon les villages. Les paysans identifient les cultivars à partir des feuilles et tiges. La productivité, le goût et la grosseur des tubercules sont les critères prioritaires de sélection. Les échanges des boutures se font entre paysans locaux $(60,84 \%)$ et par des introductions individuelles $(37,43 \%)$. Ces connaissances seront intégrées dans le programme d'amélioration variétale.

(C) 2016 International Formulae Group. All rights reserved.

Mots clés : Diversité variétale, conservation in situ, taxonomie locale, critères d'identification.

\section{Farmer management of cassava cultivars (Manihot esculenta Crantz) in the South of Chad}




\begin{abstract}
Farmers possess in their fields, many cassava cultivars which are unexplored by the research. The aim of this study is to assess their diversity and to analyze their traditional management. A total of 636 farmers selected from 43 villages, were surveyed using questionnaire and participatory rural appraisal. ShannonWeaver diversity index $(\mathrm{H})$ revealed an important level of diversity of 3.38 in the study zone. Seventy-five (75) vernacular names were recorded. Highly significant differences were observed for the number of the cultivars that ranges from 2 to 12 per village ( 6 on average). At household level, the variation is low and ranged from 1 to 4 cultivars ( 2 on average). The number of disappeared cultivars range from 1 to 14 (3.84 on average) per village. The average rate of diversity loss is $18.20 \%$ and ranging from $11 \%$ to $50 \%$ per village. Farmers use leaves and stems to identify cultivars. High yield root, taste and large sized rate were the farmer preferred criteria. The exchanges of the stems are done among local farmers $(60.84 \%)$ and by individual initiatives $(37.43 \%)$. This knowledge will be integrated in the breeding program.
\end{abstract}

(C) 2016 International Formulae Group. All rights reserved.

Keywords: Varietal diversity, in situ conservation, local taxonomy, identification criteria.

\section{INTRODUCTION}

Le manioc est la principale culture des zones tropicales et subtropicales et pratiquée essentiellement par des petites exploitations familiales. Il joue, de plus en plus, un rôle primordial dans l'alimentation des populations urbaines et rurales. C'est le deuxième aliment de base et l'une des principales sources d'énergie en Afrique sub-Saharienne (Dixon et al., 2002) mais également une ressource irremplaçable dans la lutte contre la faim et la malnutrition comme l'ont signalé certains auteurs (Nassar et Ortiz, 2010). Selon les dernières estimations de la FAO (FAOSTAT, 2014), sur 24.221.970 ha emblavés, la production mondiale est estimée à 270.293.801 t dont plus de 50\% proviennent de l'Afrique. Le Nigeria est le plus gros producteur avec 54.831.600 t/an. Au niveau des pays de la Communauté Economique et Monétaire de l'Afrique Centrale, la production annuelle moyenne sur les 5 dernières années (2010 à 2014) est de 5.699 .133 t. Le Tchad avec 240.052 t est le $5^{\text {ème }}$ producteur sur les 6 pays membres de cette zone. Actuellement, la production moyenne quinquennale est de $252.874 \mathrm{t}$ (DPSA, 2015). Les paysans détiennent un nombre important de cultivars qui sont sélectionnés et conservés pour différentes raisons et intérêts. Les travaux antérieurs sur le manioc (Elias, 2000 ; Elias et al., 2000a; Fleury, 2000 ; McKey et al., 2001 ; Pinton et Emperaire, 2001) ont mis en évidence le rôle des pratiques agricoles et sociales dans la constitution et le maintien d'une haute diversité. Cependant, ce potentiel génétique local de même que les stratégies et les méthodes de gestion paysanne n'ont pas fait l'objet d'une prospection exhaustive et de valorisation. La connaissance approfondie des facteurs socioculturels, économiques et agronomiques de cette gestion paysanne, contribue à la valorisation de ces ressources phytogénétiques locales. Dans le cas du manioc, a été mis en évidence l'importance d'une interaction entre les facteurs humains et écologiques dans la dynamique de cette diversité (Elias et al., 2000b) dont les modes de gestion reposent sur des savoirs et des savoir-faire locaux (Pinton et Emperaire, 2001). La conservation des cultivars in situ dépend de nombreux paramètres et critères qui sont propres à chaque région, ethnie où un environnement économique et socioculturel particulier. Des résultats ont ainsi été rapportés par certains auteurs et ont trait au nombre de cultivars par paysan (Emperaire et al., 2003 ; Manusset, 2006; Kombo et al., 2012). D'autres ont été évalués à l'échelle du 
village (Elias et al., 2000b; Agre et al., 2015). Les critères de sélection et de reconnaissance se réfèrent aussi bien aux caractères qualitatifs visibles que quantitatifs (Kombo et al., 2012 ; Manu-Aduening et al., 2014; Agre et al., 2016). L'attribution des noms est considérée comme un facteur culturel important dans le maintien de la biodiversité à l'échelle d'une région et certains auteurs en ont fait aussi mention en Afrique (Manu-Aduening et al., 2005 ; Delêtre, 2010 ; Koumbo et al., 2012 ; Kosh-Komba et al., 2014 ; Agre et al., 2015). Des études similaires ont été également signalées chez le niébé (Baco et al., 2008), l'oignon (Abdou et al., 2014), l'igname (Baco, 2014) et le sorgho (Sawadogo et al., 2014). Afin d'améliorer la productivité et la production du manioc et contribuer à la sécurité alimentaire au Tchad, une bonne connaissance des pratiques et savoirs traditionnels de gestion de la diversité sur cette culture est nécessaire pour l'élaboration d'un programme national d'amélioration et de création variétale. A ce jour, au niveau national, ces connaissances locales n'ont jamais été documentées. L'objectif de la présente étude est d'inventorier les cultivars de manioc, de comprendre les logiques de leur nomination, d'analyser leur étendue et distribution et d'identifier les critères de sélection et de reconnaissance utilisés par les paysans.

\section{MATERIEL ET METHODES \\ Présentation de la zone d'étude}

L'étude a été conduite dans la zone méridionale située entre le $8^{\text {ème }}$ et le $10^{\text {ème }}$ parallèle. Le climat est de type tropical semihumide, d'isohyètes $800 \mathrm{~mm}$ à plus de 1200 $\mathrm{mm}$ avec une saison sèche et une saison des pluies. Les températures moyennes varient de 24 à $38{ }^{\circ} \mathrm{C}$. Les sols sont ferrugineux lessivés de couleur rouge, de texture uniformément argilo-sableuse à argileuse avec un pH légèrement acide en surface et très acide en profondeur (Naïtormbaïdé, 2012). La végétation est caractérisée par des forêts claires et des savanes arborées dans la partie soudanienne, des forêts claires et des savanes boisées dans la zone guinéenne (DREM, 1998). Les régions, les départements, les villages et les exploitations familiales, ont été identifiés sur la base des données statistiques des rapports des campagnes agricoles (ONDR, 2013). Dans les régions de la Tandjilé et du Logone occidental, ont été choisis les départements de la Tandjilé Est et du Lac Wey. La Grande Sido et le Bahr Kôh ont été retenus dans la région du Moyen Chari. Le département des Monts de Lam a été choisi dans le Logone oriental et le Bahr Sara dans le Mandoul. Les coordonnées géographiques des villages ont été relevées à l'aide d'un GPS (Global Position System) et localisées sur une carte à l'aide du logiciel ArcGIS 10.2 (Figure 1).

\section{Méthodologie et collecte des données}

Un recensement systématique des villages où se cultive le manioc a été réalisé. Les villages au sein des différents départements ont été choisis selon la méthode de Labé et Palm (1999) en s'intéressant particulièrement aux villages de grande production de manioc. Leur nombre est compris entre 7 et 10 par département. Les paysans producteurs de manioc dans chaque village ont été également choisis quel que soit leur sexe, de façon aléatoire. Leur nombre est compris entre 12 et 16 par village, appartenant en majorité à 7 groupes ethniques. L'unité statistique est l'exploitation agricole représentée par le Chef ou l'un des membres. Dans chaque village, une rencontre préparatoire s'est d'abord tenue entre les conseillers agricoles, la recherche et les autorités locales. Une réunion générale est ensuite organisée où les objectifs et protocoles d'enquête ont été exposés. La première phase d'enquête a consisté à recueillir les informations relatives au village et de faire l'inventaire des cultivars de manioc. Cette 
collecte des données s'est faite par les méthodes de recherche participative. La seconde phase de l'enquête est une série d'entretiens structurés sous forme de questionnaire et complétés par des entretiens non structurés, menée auprès de 636 paysans.

\section{Analyses statistiques}

Les données qualitatives et quantitatives ont été analysées à l'aide du logiciel Sphinx (Sphinx Plus2-Edition LexicaV5). Des analyses de la variance (ANOVA) et des tests de Newman-Keuls au seuil de 5\% ont été effectués sur certaines données quantitatives grâce au logiciel XLSTAT-Pro version 2013.5.01. La distribution et l'étendue de la diversité variétale dans la zone d'étude ont été évaluées par la méthode d'analyse des 4 carrés ou "Four Square Analysis" utilisée par Dansi et al. (2010), décrite et utilisée sur le manioc par Kombo et al. (2012). Le taux de perte des cultivars (TPC) à l'échelle du village, a été calculé selon la méthode utilisée par Kombo et al. (2012) d'après la formule $\mathrm{TPC}=[(\mathrm{n}-\mathrm{k}) / \mathrm{N}] \times 100$ avec $\mathrm{n}$ : nombre de cultivars cultivés par peu de ménages et sur de petites superficies, $\mathrm{k}$ : nombre de cultivars nouvellement introduits et $\mathrm{N}$ : le nombre total de cultivars recensés dans le village. Les résultats ont ensuite été présentés sous forme de tableaux à l'aide du logiciel Excel version 2007. Le degré de la diversité des cultivars dans la zone d'étude a été déterminé par l'indice de diversité (H) selon Shannon (1948). Cet indice a été utilisé par Agre et al. (2015) pour évaluer l'importance de la diversité variétale du manioc.

\section{RESULTATS \\ Profil des enquêtés}

Les paysans enquêtés sont en majorité des hommes (92\%) et peu de femmes $(8 \%)$. En effet, au Tchad, la culture du manioc est essentiellement pratiquée par des hommes. Les femmes sont plutôt fortement impliquées dans les opérations post récolte. Le christianisme est la religion la plus pratiquée avec en majorité des protestants $(60 \%)$ et un peu moins de catholiques (37\%). L'âge moyen des enquêtés est de 38 ans dont des jeunes $(42,90 \%)$ et de personnes de plus de 35 ans $(57,10 \%)$. Leur niveau d'études est moyen, primaire et secondaire $(85 \%)$ et peu d'analphabètes (11\%). Le nombre moyen par ménage est de 10 personnes avec 11 ans d'expérience dans la culture du manioc. Sur le plan linguistique, 18 groupes ethniques ont été identifiés et les dialectes les plus parlés sont le NGambaye, le Lakka, le Mboum, le Zimé, le Mbaye et le Sara madjingaye.

\section{Importance de la culture du manioc et usages}

Dans la zone d'étude, chez les exploitations productrices de manioc, la culture du manioc $(36,30 \%)$ est la première des trois cultures les plus pratiquées avec l'arachide $(23,30 \%)$ et le sorgho $(22,50 \%)$. Le manioc est cité en première position dans la région du Mandoul (20,80\%), en deuxième position dans les deux Logones et le Moyen Chari (14,40\%). La moyenne des superficies emblavées est de 1,75 ha par paysan. Plus de la moitié des paysans enquêtés $(56,30 \%)$ cultivent du manioc sur des superficies comprises entre 1 et 3 ha. Très peu $(15,50 \%)$ le font sur des superficies supérieures à 3 ha alors que certains $(28,20 \%)$ n'utilisent que de petits champs de moins d'un ha. Les différentes parties de la plante sont utilisées à différents fins. Les tubercules sont destinés à la fois à l'autoconsommation et à la vente estiment la majorité des paysans enquêtés (92\%). Concernant les feuilles même si certains enquêtés $(47,00 \%)$ déclarent les vendre, un peu plus de la moitié $(52,50 \%)$ affirment qu'ils consomment directement une bonne partie de leur production. Quant aux tiges, elles sont soit réutilisées comme boutures dans les nouveaux champs $(68,40 \%)$, directement vendues $(67,50 \%)$, soit utilisées 
comme bois de chauffe $(29,70 \%)$. Une faible proportion $(6,50 \%)$ les utilise dans l'alimentation du bétail tandis que d'autres $(3,60 \%)$ affirment les utiliser dans la production du sel local. A peine 2,50\% disent les abandonner dans les champs où les bruler. Sur le plan technologique, les feuilles et les tubercules sont aussi souvent transformées sous diverses formes et selon les ethnies. La transformation des feuilles, principale activité féminine, est pratiquée par de nombreux ménages (80,80\%). Elles sont séchées, blanchies puis cuites avec d'autres ingrédients sous forme de sauce. Dans le cas des tubercules, les ménages sont encore plus nombreux à les transformer $(94,30 \%)$. Les produits élaborés les plus courants sont les cossettes (50\%), le mamberé (44,70\%) qui est une sorte de pâte de manioc enroulée dans des feuilles de bananier ou de mil et cuite à la vapeur, les tubercules fraîches cuites $(43,90 \%)$ et la pâte $(42,60 \%)$.Très peu de paysans produisent de l'amidon $(13,40 \%)$ et du gari $(6,90 \%)$. Les ethnies les plus impliquées dans la production de l'amidon sont les Lakka $(58,96 \%)$ et pour le gari ce sont les Kaba (31,88\%). Les Mbaye font souvent cuire les tubercules doux $(60,85 \%)$, produisent de la pâte $(28,30 \%)$ et du mamberé $(60,85 \%)$. La transformation en cossettes, pour la conservation et la production ultérieure de la farine, est une activité surtout présente en territoire Ngambaye $(24,44 \%)$ et Lakka $(21,21 \%)$.

\section{Diversité variétale du manioc dans la zone d'étude}

Taxonomie locale et logiques paysannes d'attribution des noms: Sur les 5 sites enquêtés, 75 noms vernaculaires ont été inventoriés. Il en ressort que, 5 portent leurs noms d'origine, 7 correspondent aux noms des villages, 4 autres sont des prénoms et 2 font référence à la structure de recherche qui les a introduit. Les appellations sont très variées avec quelquefois des synonymies dans d'autres groupes ethniques. Les faits socioculturels, les avantages que procure la culture du manioc, les origines, le cycle, le goût et les différentes parties de la plante sont utilisés par les paysans pour nommer les cultivars. Cinq cultivars sont d'origine centrafricaine et dont les noms ont des significations identiques à certains noms locaux. Les cultivars les plus cités sont Tessem (38,70\%), Six mois $(17,50 \%)$, Boyo $(15,40 \%)$, Pangassou $(14,60 \%)$ et Tamadoz $(13,50 \%)$.

Diversité des cultivars à l'échelle de la région, du village et des ménages: Le nombre de cultivars inventoriés varie de 2 à 12 par village avec une moyenne de 6 sur l'ensemble de la zone d'étude (Tableau 1). Les différences significatives observées au seuil de $5 \%$ pour le nombre de cultivars par village ont permis de les classer en deux groupes bien distincts. C'est dans les régions du Mandoul et du Logone occidental, avec une moyenne de 8 cultivars par village, qu'a été enregistrée la plus forte diversité avec 11 et 12 cultivars. La plus faible diversité, 2 et 3 cultivars, a été observée dans les régions du Logone oriental, du Moyen Chari et de la Tandjilé avec respectivement en moyenne: 3,$89 ; 4,90$ et 5,57 de cultivars par village. Les régions à forte diversité variétale, sont peuplées des Mbaye. Quant aux trois autres régions, elles sont habitées en majorité des Lakka et Mboum (Logone oriental), des Saramadjingaye (Moyen Chari), des Zimé et Lele (Tandjilé). A l'échelle des ménages, le nombre de cultivars varie de 1 à 4 avec une moyenne de 2 cultivars par ménage. La majorité des ménages enquêtés $(65,88 \%)$ utilisent chacun 2 à 3 cultivars. Environ un quart $(24,69 \%)$ utilisent seulement 1 cultivar par ménage. Ceux qui détiennent le plus grand nombre, soit 4 cultivars par ménage, sont 
moins nombreux $(9,43 \%)$ et tous originaires des villages du département du Bahr Sara peuplé en majorité des Mbaye. Par ailleurs, l'indice de diversité de Shannon obtenu est de 3,38 pour toute la zone d'étude.

Evaluation du taux de perte des cultivars: A l'échelle du village et sans tenir compte des synonymies, ce sont 38 cultivars qui ont disparu. Ce nombre varie de 1 à 14 avec en moyenne 3,81 cultivars par village. En effet, 11 villages totalisent entre 6 et 8 cultivars et 12 autres entre 3 et 5 cultivars disparus. Dans 7 villages enquêtés, seulement 1 et 2 cultivars sont signalés comme disparus. Même si aucune disparition n'a été observée dans 10 villages, ce phénomène est très prononcé dans les villages Sateignan II, Sateignan I et Koudoti avec respectivement 11,13 et 14 cultivars (Tableau 2). Par ailleurs, la moyenne de cultivars utilisés par beaucoup de ménages sur de grandes superficies n'est qu'un seul cultivar. Par contre, cette moyenne est 2 cultivars pour la catégorie correspondant aux petites superficies emblavées par peu de paysans. Le taux moyen de perte des cultivars pour toute la zone d'étude, est de $18,20 \%$ et varie entre $11 \%$ dans le village de Bira II et $50 \%$ dans les villages Kokante, Dogou Yatreo et Koumao. Ce taux est nul dans 30,23\% des villages de la zone d'étude. Au niveau régional, le nombre de cultivars disparus varie de 4 à 21 avec en moyenne 11,20 cultivars. Les régions du Mandoul et du Logone oriental avec respectivement 21 et 14 cultivars sont les plus touchées. Seulement 4 cultivars identifiés dans le village de Daradja ont disparu de la région de la Tandjilé pour 8 dans le Moyen Chari et 9 dans le Logone occidental.

Importance de la distribution et de l'étendue des cultivars : Cette distribution ne concerne que les cultivars présents dans au moins 3 villages (Tableau 3). Les cultivars Tessem et Simon ont une large distribution car présents respectivement dans 27 et 22 villages. Les cultivars Mandrakako, Mbogueral, Lizette et Sima sont cultivés uniquement sur de petites superficies et par peu de ménages. Au total, $55 \%$ de cultivars sélectionnés sont emblavés par beaucoup de ménages sur de grandes superficies. Les cultivars Delila, Ndabodleub, Djihoko, Konhongo, Bandou, Ngalindah et Damain ne sont présents que dans 3 villages et utilisés par peu de ménages et sur de grandes superficies.

\section{Critères paysans d'identification et de préférence des cultivars}

Ces critères sont soit uniques soit une combinaison de plusieurs autres. Les résultats montrent que les paysans $(70,10 \%)$ reconnaissent les cultivars à partir de leurs feuilles. D'autres $(40,80 \%)$ le font à partir des tiges. Les pétioles $(2,70 \%)$ et les tubercules $(3,30 \%)$ sont très peu utilisés comme critères. Toutefois, d'autres critères dits complémentaires comme la couleur, la forme, la taille, le nombre de lobes et le nombre de ramifications, permettent aux paysans de compléter leur système d'identification. Quant à la sélection des cultivars, celle-ci repose sur 9 critères de type agronomique, technologique et économique. Les 3 critères prioritaires de choix des cultivars sont respectivement la productivité, le goût et la grosseur des racines (Tableau 4). La précocité, la facilité à la transformation, les revenus générés et la résistance aux maladies et ravageurs sont aussi des critères jugés importants. Seuls 5\% des enquêtés ont retenu la tolérance à la sècheresse. Au niveau des régions, pour les paysans de la Tandjilé $(13,40 \%)$, seule la productivité est le critère le plus déterminant. Les paysans du Mandoul (21,90\%) et du Moyen Chari $(16,00 \%)$ sont plutôt intéressés par les cultivars résistants aux ravageurs, aux maladies ainsi qu'à leur productivité. La répartition de ces critères sur le plan ethnique montre des niveaux très divers (Tableau 5). 
Chez les Lakka, Ngambaye et Mboum, 7 critères de préférence ont été dénombrés pour 9 chez les Mbaye et les Sara.

\section{Modes d'obtention des boutures}

Les principaux modes d'obtention des boutures dans la zone d'étude sont les échanges du matériel génétique entre paysans d'une même localité $(60,84 \%)$ et des introductions faites par quelqu'un du village $(37,43 \%)$. Seulement quelques paysans interrogés $(26,10 \%)$ affirment que cette introduction se fait à travers les institutions de recherche et de vulgarisation. Les résultats par région (Tableau 6) montrent que les échanges locaux sont élevés dans les régions du Mandoul $(36,43 \%)$ et du Moyen Chari (29,46\%). De même, les paysans du Mandoul $(38,66 \%)$ et ceux du Moyen Chari $(32,35 \%)$ signalent que les cultivars sont introduits par quelqu'un du village. Les implications de la recherche et des services de vulgarisation ont été signalées dans le Mandoul (31,33\%) et dans le Logone oriental (31,93\%). Dans le Mandoul, la majorité des paysans $(59,78 \%)$ rapportent que les boutures sont introduites par les organisations non gouvernementales alors que dans la Tandjilé, un nombre plus élevé $(82,14 \%)$ estime que cela se fait à travers les projets.

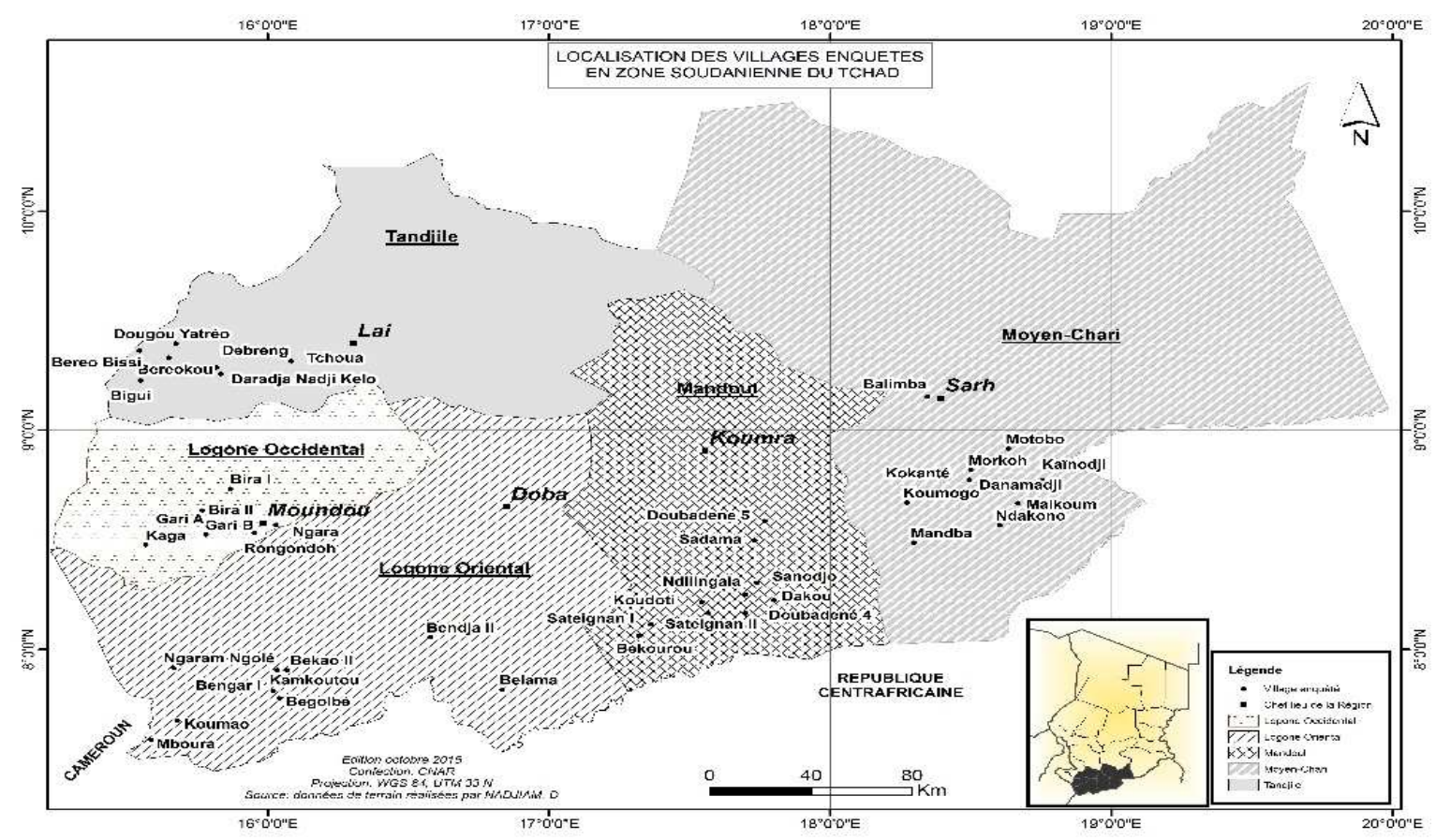

Figure 1: Carte de localisation géographique des régions et des villages enquêtés. 
Tableau 1: Variation de la diversité du manioc à l'échelle des régions et villages.

\begin{tabular}{|c|c|c|c|c|c|}
\hline Régions & $\begin{array}{c}\text { Ethnies } \\
\text { majoritaires }\end{array}$ & $\begin{array}{c}\text { Nombre } \\
\text { de villages }\end{array}$ & $\begin{array}{l}\text { Valeur minimum } \\
\text { de cultivars }\end{array}$ & $\begin{array}{c}\text { Valeur maximum } \\
\text { de cultivars }\end{array}$ & $\begin{array}{c}\text { Nombre moyen } \\
\text { de cultivars }\end{array}$ \\
\hline Logone oriental & Lakka/MBoum & 9 & 2 & 6 & $3,89 \pm 0,71 \mathrm{a}$ \\
\hline Moyen Chari & Saramadjingaye & 10 & 2 & 9 & $4,90 \pm 0,68 \mathrm{a}$ \\
\hline Tandjilé & Zimé/Lele & 7 & 3 & 8 & $5,57 \pm 0,81 \mathrm{a}$ \\
\hline Mandoul & Mbaye & 10 & 4 & 11 & $7,90 \pm 0,68 b$ \\
\hline Logone Occidental & Ngambaye & 7 & 5 & 12 & $8,57 \pm 0,81 \mathrm{~b}$ \\
\hline Zone d'étude & & 43 & 2 & 12 & $6,16 \pm 0,74$ \\
\hline
\end{tabular}

Tableau 2: Taux de perte de cultivars de manioc par village.

\begin{tabular}{|c|c|c|}
\hline Villages & $\mathbf{M}^{-} \mathbf{S}^{-}$ & T.P. $(\%)$ \\
\hline Belama, Kaïnodji, Morkoh, Balimba, Madimba, Danamadji & 0 & 0 \\
\hline Kamkoutou, Bendja II, Mboura, Ngaramngolé, Tchoua, Bere & 1 & 0 \\
\hline Doubadene IV & 3 & 0 \\
\hline Bira II & 5 & 11 \\
\hline Sanodjo, Sateignan II & 2 & 12,5 \\
\hline Debreng & 2 & 14,29 \\
\hline Berokouh, Sateignan I, Dakou & 2 & 16,67 \\
\hline Bengar I & 3 & 16,67 \\
\hline Doubadene V & 2 & 18,18 \\
\hline Bigui, Motobo, Koudoti & 2 & 20 \\
\hline Ngara, Gari A, Sadama & 3 & 20 \\
\hline Ndakono & 2 & 22,22 \\
\hline Maïkoum, Koumogo, Ndilingala & 1 & 25 \\
\hline Begolbé & 2 & 25 \\
\hline Gari B & 5 & 28,57 \\
\hline Bekao II & 3 & 33,33 \\
\hline Rogondoh & 4 & 33,33 \\
\hline Bekourou & 5 & 36,36 \\
\hline Daradja & 4 & 37,5 \\
\hline Kaga & 6 & 41,67 \\
\hline Bira I & 7 & 44,44 \\
\hline Koumao, Kokante & 1 & 50 \\
\hline DogouYatréo & 4 & 50 \\
\hline Moyenne pour la zone d'étude & 2 & 18,2 \\
\hline
\end{tabular}


Tableau 3 : Distribution et étendue des cultivars observées dans au moins 3 villages.

\begin{tabular}{|c|c|c|c|c|c|}
\hline Noms locaux des cultivars & NTV & $\mathbf{M}^{+} \mathbf{S}^{+}$ & $\mathbf{M}^{+} \mathbf{S}^{-}$ & $\mathbf{M}^{-} \mathbf{S}^{+}$ & $\mathbf{M}^{-} \mathbf{S}^{-}$ \\
\hline Tessem & 27 & 11 & 6 & 3 & 7 \\
\hline Poum Poum & 9 & 1 & 0 & 4 & 4 \\
\hline Rendah & 5 & 1 & 0 & 2 & 2 \\
\hline Simon & 22 & 4 & 1 & 7 & 10 \\
\hline Mandrakako & 7 & 0 & 0 & 0 & 7 \\
\hline Tinodji & 10 & 1 & 0 & 5 & 4 \\
\hline Mbogueral & 5 & 0 & 0 & 0 & 5 \\
\hline Mbindakass & 4 & 0 & 0 & 2 & 2 \\
\hline Ndjabra & 3 & 0 & 0 & 1 & 2 \\
\hline Moreye & 6 & 1 & 3 & 2 & 0 \\
\hline Ngalibaka & 5 & 2 & 1 & 1 & 1 \\
\hline Lizette, Sima ${ }^{(1)}$ & 3 & 0 & 0 & 0 & 3 \\
\hline Madjingani & 6 & 0 & 0 & 4 & 2 \\
\hline Kangaba & 11 & 2 & 0 & 4 & 5 \\
\hline Pangassou & 9 & 7 & 0 & 0 & 2 \\
\hline Mandoz & 8 & 4 & 0 & 1 & 3 \\
\hline Ngouôkoh & 5 & 1 & 0 & 1 & 3 \\
\hline Воуо & 10 & 6 & 0 & 1 & 3 \\
\hline Guebida & 5 & 4 & 0 & 0 & 1 \\
\hline Bebedjia, Tamandodji, Tolmad ${ }^{(2)}$ & 4 & 1 & 0 & 3 & 0 \\
\hline Karanga & 3 & 0 & 0 & 2 & 1 \\
\hline Gentil & 3 & 1 & 0 & 2 & 0 \\
\hline Madinta & 3 & 2 & 0 & 1 & 0 \\
\hline Delila,Ndabodleub, Djihoko, Konhongo ${ }^{(3)}$ & 3 & 0 & 0 & 3 & 0 \\
\hline \multicolumn{6}{|l|}{ Bandou, Ngalindah, Damain } \\
\hline
\end{tabular}

Tableau 4: Principaux critères paysans de sélection variétale du manioc dans le sud du Tchad.

\begin{tabular}{lc}
\hline Types de critères & Réponses des producteurs $(\boldsymbol{\%})$ \\
\hline Productivité & $\mathbf{8 9 , 8 0}$ \\
Goût (doux) & $\mathbf{7 8 , 8 0}$ \\
Grosses tubercules & $\mathbf{7 2 , 8 0}$ \\
Précocité & 63,40 \\
Facilité de transformation & 53,80 \\
Forte valeur marchande & 51,60 \\
Résistance aux maladies & 51,30 \\
Facilité de cuisson & 35,40 \\
Tolérance à la sècheresse & 4,90 \\
\hline
\end{tabular}


Tableau 5: Critères de sélection des cultivars selon les groupes ethniques majoritaires et pourcentages de réponses correspondants à chaque critère donné.

\begin{tabular}{|c|c|c|c|c|c|c|}
\hline \multirow{2}{*}{ Critères de sélection } & \multirow{2}{*}{$\begin{array}{c}\text { Pourcentage de } \\
\text { réponses dans la zone } \\
\text { d'étude }\end{array}$} & \multicolumn{5}{|c|}{$\begin{array}{l}\text { Pourcentage de réponses } \\
\text { par groupe ethnique majoritaire }\end{array}$} \\
\hline & & Lakka & Ngambaye & Mboum & Mbaye & ara \\
\hline Productivité & 89,80 & 9,40 & 13,10 & 5,80 & 28,00 & 4,90 \\
\hline Goût (doux) & 78,80 & 7,70 & 10,70 & 5,80 & 27,40 & 4,70 \\
\hline Grosses tubercules & 72,80 & 7,90 & 9,80 & 3,60 & 27,40 & 4,40 \\
\hline Précocité & 63,40 & 9,40 & 9,60 & 6,10 & 26,30 & 3,30 \\
\hline Facilité de transformation & 53,80 & 3,60 & 5,20 & 3,50 & 26,70 & 2,50 \\
\hline Résistance aux maladies & 51,30 & 0,30 & 5,40 & 1,40 & 26,10 & 3,90 \\
\hline Forte valeur marchande & 51,60 & 6,50 & 6,50 & 1,30 & 15,40 & 0,50 \\
\hline Facilité de cuisson & 35,40 & 2,00 & 7,10 & 2,50 & 14,60 & 0,60 \\
\hline Tolérance à la sécheresse & 4,90 & - & - & - & 4,40 & 0,20 \\
\hline
\end{tabular}

Tableau 6: Modes d'obtention des boutures par région d'étude.

\begin{tabular}{lccccc}
\hline Régions & Localement & $\begin{array}{c}\text { Mode d'obtention des boutures } \\
\text { Quelqu'un } \\
\text { du village }\end{array}$ & $\begin{array}{c}\text { Recherche/ } \\
\text { vulgarisation (\%) }\end{array}$ & ONG & Projet \\
\hline Mandoul & 36,43 & 38,66 & 31,33 & 59,78 & 3,57 \\
Moyen Chari & 29,46 & 32,35 & 10,24 & 11,96 & 7,14 \\
Logone Oriental & 4,65 & 17,23 & 31,93 & 28,26 & - \\
Tandjilé & 16,80 & 5,88 & 5,42 & - & 82,14 \\
Logone occidental & 12,66 & 5,88 & 21,08 & - & 7,14 \\
Total & 100 & 100 & 100 & 100 & 100 \\
\hline
\end{tabular}

\section{DISCUSSION}

Dans le Sud du Tchad, le manioc est de plus en plus cultivé en association avec d'autres plantes. Cette stratégie a eu des impacts positifs sur les revenus des producteurs comme cela fut rapporté également dans une étude conduite au Nigeria où l'association du manioc avec l'igname, le maïs et le melon a été très bénéfique en termes de gains financiers (Obasi et al., 2015). L'importance du manioc dans cette étude a été aussi révélée par les usages variés de ses différentes parties. Les tubercules et les feuilles sont destinés à la consommation et à la vente. Les tiges sont de plus en plus valorisées comme boutures, bois de chauffe et pour la production d'un sel local destiné surtout à des usages médicinaux. Les feuilles sont consommées après blanchiment et cuisson. Cependant, les produits dérivés de la transformation de ces feuilles sont peu variés et diversement appréciés selon les localités. Par rapport aux tubercules, l'étude a révélé plusieurs produits dérivés dont le mamberé d'origine centrafricaine, l'amidon et le gari introduits à partir du Nigeria.

Le nom local le plus largement répandu du manioc au Tchad est «ngali ou ngari ». L'attribution des noms vernaculaires aux cultivars ainsi que leurs significations sont des pratiques courantes ancrées dans les sociétés traditionnelles. Ces pratiques ont été signalées dans plusieurs travaux. Missihoun et al. (2012) ont affirmé que le nom local est l'unité de base que les producteurs utilisent dans la gestion et la sélection des ressources 
végétales. L'importance de ce facteur culturel dans la gestion et le maintien de la biodiversité a été également rapportée par Manusset (2006). Dans la présente étude, pour nommer les cultivars, les paysans utilisent des noms des personnes et des villages d'origine, des similarités avec d'autres plantes, des proverbes et des expressions relatives aux avantages que génère la culture du manioc surtout pendant les périodes de soudure ou de famine. Les populations amérindiennes, accordent quant à eux, plus d'importance aux traits architecturaux et le cultivar est nommé en se référant à la partie épigée de la plante (Emperaire et al., 2003). Au Tchad, quand un cultivar n'a pas un nom spécifique, il est tout simplement qualifié de manioc doux ou amer dans les différents dialectes. Les qualificatifs doux les plus répandus sont "koul ", "sohol ", "moreye" et pour les cultivars amers "ade", "maguilira" et "ate". Ce qui n'est pas le cas en Amazonie où les variétés douces sont regroupées sous un seul nom de macaxeiras dans les régions Centre et Nord-Est du Brésil et les variétés amères sont nommées mandiocas dans la partie Nord-Ouest (Emperaire et al., 2003). Par ailleurs, chez les Wayãpi de la Guyane française, à un cultivar donné de manioc peut être attribué plusieurs noms qui sont souvent des types de combinaisons motivées (Grenand, 2002). Ces stratégies paysannes de nomination des cultivars sont identiques à celles observées dans cette zone d'étude et l'analyse révèle que les Ngambaye et les Mbaye attribuent plus de noms aux cultivars avec respectivement 21 et 13 dénominations. Ces modes de nomination ont été aussi signalés sur des variétés de sorgho à grains sucrés du Burkina par Sawadogo et al. (2014) et aussi sur le manioc au Vanuatu par Sardos et al. (2008). Dans le sud du Tchad, la langue de l'ethnie majoritaire et originaire de la région est utilisée pour nommer les cultivars locaux ou renommer ceux qui sont introduits. Dans ce dernier cas, ne sont pas renommés les cultivars provenant d'un même ou proche groupe linguistique comme cela a été signalé dans une étude sur les taros au Vanuatu (Caillon et al., 2005). Contrairement à Baco (2014) qui estime que les transferts variétaux de l'igname au Bénin, s'accompagnent rarement de transferts de nom.

La plupart des cultivars inventoriés sont locaux et leur nombre est faible, comparé à d'autres zones. Chez les amérindiens Makushi, ont été dénombrées 76 variétés de manioc (Elias, 2000). Dans toute la Guyane française, cette diversité variétale est encore plus élevée avec 296 variétés recensées (Manusset, 2006). Malgré cette différence, le potentiel génétique local mis en évidence dans cette étude, est important. L'indice de Shannon est aussi important que celui obtenu dans le cas de l'étude des cultivars de manioc du Centre Bénin et qui traduit une forte diversité variétale (Agre et al., 2015). L'inventaire a aussi révélé que c'est un cultivar amélioré doux qui est le plus cultivé. Cependant, il n'existe pas une répartition particulière de types de cultivars en fonction des régions. Les populations du sud du Tchad, tout comme ceux de la partie basse du Bassin Amazonien (Bradbury et al., 2013) cultivent à la fois des cultivars doux et amers contrairement en Amérique du Sud où beaucoup de groupes d'indigènes de l'Ouest d'Amazonie ne cultivent que du manioc doux. Cependant, aussi bien à l'échelle du village que des ménages, cette diversité est inférieure par rapport aux résultats obtenus au Congo (Kombo et al., 2012). Toutefois, le nombre de cultivars utilisés par ménage est similaire à celui obtenu au Ghana, (Manu-Aduening et al., 2005). Par contre, il est supérieur à celui observé dans le Sud et Centre Benin (Agre et al., 2016). Par rapport aux villages amérindiennes à forte diversité (Emperaire et al., 2003), il est plutôt très faible. Le nombre élevé des variétés observé dans les champs des Amérindiens s'explique par le fait que ces derniers combinent le système de propagation par voie végétative et sexuée (Sardos et al., 2008; McKey, 2012). Ces pratiques ont un impact important sur la dynamique du maintien de la diversité (Elias et al., 2001 ; 
Sardos et al., 2008; McKey et al., 2012). Dans le Centre du Benin, la multiplication du manioc à partir des graines est peu connue des paysans (Agre et al., 2015). Au Tchad, la culture du manioc se pratique uniquement sous forme de boutures. Afin de conserver leurs cultivars, les paysans les gardent jalousement en les replantant, ce qui explique en partie, le faible nombre de cultivars observé au niveau des ménages. L'étude a aussi révélé que des variations sur le nombre de cultivars ont été observées entre villages d'une même région et même entre les villages des régions différentes. Les détenteurs d'un ou plusieurs types de cultivars dans un village, sont bien connus. Ces résultats sont similaires aux travaux de Willemen et al. (2007), qui affirment que les paysans ont vraiment conscience du nombre exact et précis des cultivars existant dans leurs villages. La prise en compte du nombre de cultivars à l'échelle du village pour évaluer la diversité variétale, a été aussi rapportée sur d'autres spéculations (Missihoun et al., 2012; Gbaguidi et al., 2013). La diversité observée au niveau des villages et par rapport aux groupes ethniques, montre que la culture du manioc est appréhendée de manière différente selon les communautés. En milieu Mbaye et Ngambaye, on trouve plus de cultivars que dans les autres villages de la zone d'étude. Ce sont aussi les deux ethnies qui attribuent plus de noms aux cultivars. Ce qui n'est pas le cas de la Tandjilé composée en majorité des Zimé et des Lele, qui est plutôt une zone traditionnelle de la culture du riz.

Le taux moyen de perte, à cause des contraintes aussi bien agronomiques que technologiques, est relativement important et même très élevé dans certains villages. Comparé à certaines études antérieures (Kombo et al., 2012 ; Agre et al., 2015), ce taux est moyen. Malgré ces contraintes, les cultivars continuent à être conservés pour des besoins spécifiques.

La distribution et l'étendue des cultivars varient d'un village à l'autre et d'une région donnée à une autre. Cette inégale répartition et localisation géographique est due au faible transfert des boutures entre localités éloignées, à l'adaptabilité de certains cultivars à une région, aux choix des paysans et à la proximité des zones d'introduction. Par rapport au risque de leur disparition totale qui est confirmée par le fait que moins d'un quart sont cultivés sur de grandes superficies et par beaucoup de ménages, il serait souhaitable qu'ils soient intégrés dans un programme national de sélection variétale. En effet, Elias et al. (2000b) ont montré que les cultivars locaux de manioc constituent une source non négligeable en matière de diversité génétique.

Dans la zone d'étude, les paysans utilisent un ou plusieurs traits pour reconnaître les cultivars. Les feuilles et les tiges sont plus utilisées et elles constituent le premier niveau d'identification car elles sont facilement observables dans les champs comme l'ont également rapporté Agre et al. (2016). Les caractères liés aux tubercules serviront dans une seconde phase à compléter les informations obtenues au niveau des parties aériennes. Ce qui n'est pas le cas du CentreNord de la Côte d'Ivoire où les paysans identifient les cultivars de maïs exclusivement à l'aide d'un seul trait qui est la coloration des grains (N'Da et al., 2013). Pour la sélection des cultivars, les paysans utilisent trois critères prioritaires, la productivité, le goût et la grosseur des tubercules. Ces critères sont identiques à ceux rapportés par Kombo et al. (2012). Mais leur importance est très variable car les contraintes de la culture du manioc se posent différemment selon les localités.

Quant aux modes d'obtention des boutures, les mêmes boutures passent d'une exploitation à l'autre. Ce fait a été aussi rapporté par Abdou et al. (2014) qui affirment que la gestion de la diversité des ressources génétiques de l'oignon est affectée par les modes d'échange, de transmission et de sélection de semences. Différents modes d'échanges ont été aussi signalés dans le cas du niébé (Baco et al., 2008), du manioc (Mckey et al., 2001) et de l'igname (Baco, 2014). Dans la présente étude, les boutures 
sont souvent échangées, offertes gratuitement et parfois vendues. Au niveau des régions, les échanges inter paysans sont plus importants dans les régions du Mandoul et du Moyen Chari. Le faible niveau d'introduction des boutures à partir des institutions spécialisées, est à l'origine du faible nombre de cultivars améliorés recensés. Dans le Logone occidental, ces échanges et introductions sont les plus faibles.

\section{Conclusion}

L'étude a montré que bien que la culture du manioc soit récente, celle-ci, de par l'importance de sa production, contribue de manière significative à la sécurité alimentaire. Les multiples usages des différentes parties de cette plante ainsi que les nombreux produits dérivés issus des transformations technologiques et culinaires montrent l'intérêt de cette culture pour les populations. La diversité nommée est significative dans la zone d'étude. Les stratégies paysannes de maintien de cette diversité sont basées sur la conservation in situ. Les cultivars sont caractérisés par des noms vernaculaires qui permettent d'avoir des informations sur l'origine, le cycle, les rendements, les caractéristiques technologiques et organoleptiques, la couleur, la forme et l'aspect des différentes parties de la plante. Ils sont sélectionnés en fonction des besoins spécifiques des paysans et des groupes ethniques. Des critères sont utilisés pour leur identification et les échanges des boutures se font surtout entre paysans. Ces pratiques ont un impact important sur cette diversité. Même si les paysans connaissent bien le niveau de diversité dans leur village et les environnants, le taux élevé de perte des cultivars et leur méconnaissance de la reproduction sexuée, constituent des risques réels non seulement sur cette diversité mais aussi sur la culture du manioc. Par conséquent, l'encadrement des paysans dans l'utilisation de la reproduction sexuée et les analyses moléculaires des cultivars identifiés dans cette étude, devront faire partie du programme national de recherche.

\section{CONFLITS D'INTERETS}

Les auteurs déclarent qu'il n'y a pas de conflits d'intérêts entre eux.

\section{CONTRIBUTIONS DES AUTEURS}

DN a conduit l'enquête sur le terrain, collecté les données auprès des producteurs, analysé, interprété les résultats et préparé le manuscrit. MD a contribué à l'identification des producteurs et des régions d'enquête, l'interprétation des résultats mais aussi à la préparation du présent manuscrit. JMMM a supervisé le déroulement de l'enquête, participé à l'identification des bassins de production du manioc et apporté des corrections au manuscrit. AG a supervisé de manière constante toutes les activités planifiées, examiné les résultats, analysé et donné des orientations, préparé et apporté des corrections au manuscrit.

\section{REMERCIEMENTS}

Nous exprimons notre profonde reconnaissance au Délégué National du PRASAC, Brahim Hissene HIREKE, à Wedjou KEBGNABE et aux appuis techniques de Ehnon GONGNET, Djim-assal DATOLOUM et Mahamat Le DIAMBO.

\section{REFERENCES}

Abdou R, Malice M, Bakasso Y, Saadou M, Baudoin JP. 2014. Taxonomie locale et analyse des critères des paysans pour caractériser les différents écotypes d'oignons (Allium cepa L.) du Niger. Cah. Agric., 23(3): 166-176. DOI: 10.1684/agr.2014.0700

Agre AP, Kouchade S, Odjo T, Dansi M, Nzobadila B, Assogba P, Dansi A, Akoegninou A, Sanni A. 2015. Diversité et évaluation participative des cultivars du manioc (Manihot esculenta Crantz) au Centre Benin. Int. J. Biol. 
Chem. Sci., 9(1): 388-408. DOI: http://dx.doi.org/10.4314/ijbcs.v9i1.33

Agre AP, Badara G, Adjatin A, Dansi A, Bathacharjee R, Rabbi IY, Dansi M, Gedil M. 2016. Folk taxonomy and traditional management of cassava (Manihot esculenta Crantz) diversity in southern and central Benin. International Journal of Innovation and Scientific Research, 20(2): 500-515.

Baco MN, Ahanchedé A, Bello S, Dansi A, Vodouhè R, Biaou G, Lescure JP. 2008. Evaluation des pratiques de gestion de la diversité du niébé (Vigna unguiculata): une tentative méthodologique expérimentée au Bénin. Cah. Agric., 17(2):183-188. DOI:10.1684/agr.2008.0169

Baco MN. 2014. Les pratiques de gestion de la diversité variétale de l'igname au Bénin. Agridape, 30(1): 3.

Bradbury EJ, Duputié A, Delêtre M, Roullier C, Narvaez-Trujillo A, Manu-Aduening JA, Emshuiller E, McKey D. 2013. Geographic differences in patterns of genetic differenciation among bitter and sweet manioc (Manihot esculenta subsp. esculenta; Euphorbiaceae). American Journal of Botany, 100(5): 857-866. DOI:10.3732/ajb. 1200482

Caillon S, Degeorges V, Lanouguère-Bruneau P, Lebot V, Quero-García J. 2005. Les taros du Vanuatu: que conserver et comment? Natures Sciences Sociétés, 13: 306-310.

Dansi A, Adoukonou-Sagbadja H, Voduhe R. 2010. Diversity, conservation and related wild species of Fonio millet (Digitaria spp.) in the northwest of Benin. Genet. Resour. Crop. Evol., 57: 827-839.

Delêtre M. 2010. The ins and outs of manioc diversity in Gabon, Central Africa. A pluridisciplinary approach to the dynamics of genetic diversity of Manihot esculenta Crantz (Euphorbiaceae). $\mathrm{PhD}$ thesis,
University of Dublin, Trinity College, p.364.

Direction de la production et des statistiques (DPSA) agricoles. 2015. Rapport du Tchad. Concertation régionale sur la situation alimentaire et nutritionnelle au Sahel et en Afrique de l'Ouest. Nouackchott du 01-03 avril 2015, p.26.

Direction des ressources en eau (DREM) et de la météorologie. 1998. Carte climatique et formations végétales du Tchad, p.1.

Dixon AGO, Ngeve JM, Nukenine EN. 2002. Response of cassava genotypes to four biotic constraints in three agroecologies of Nigeria. Afr. Crop Sci. J., 10(1): 11-21.

Elias M. 2000. Le manioc chez les Makushi: une richesse à valoriser. In les Peuples des Forêts Tropicales Aujourd'hui (vol. 4), Grenand P (ed). Région Caraïbes Guyanes Bélize Programme APFT: Bruxelles; 201-221.

Elias M, Panaud O, Robert T. 2000a. Assessment of genetic variability in a traditional cassava (Manihot esculenta Crantz) farming system, using AFLP markers. Heredity, 85: 219-230.

Elias M, Rival L, McKey D.2000b. Perception and management of cassava (Manihot esculenta, Crantz) diversity among Makushi Amerindians of Guyana (South America). Journal of Ethobiology, 20(2): 239-265.

Elias M, Penet L,Vindry P, McKey D, Panaud O, Robert T. 2001.Unmanaged sexual reproduction and the dynamics of genetic diversity of a vegetative propagated crop plant, cassava (Manihot esculenta), in a traditional farming system. Mol. Ecol., 10: 18951907.

Emperaire L, Santos Mühlen G, Fleury M, Robert T, Mckey D, Pujol B, Elias M. 2003. Approche comparative de la diversité génétique et de la diversité morphologique des maniocs en Amazonie (Brésil et Guyanes). Les Actes du BRG, 4: 247-267. 
FAOSTAT. 2014. Base de données statistiques agricoles. Rome, Italie. http://faostat3.fao.org; Consulté le 24/12/2015.

Fleury M. 2000. L'agriculture Wayana: une transition viable. In: Les peuples des Forêts Tropicales Aujourd'hui, Grenand P (ed). Région Caraïbes Guyanes Bélize Programme APFT: Bruxelles ; 151-161.

Gbaguidi AA, Dansi A, Loko LY, Dansi M, Sanni A. 2013. Diversity and agronomic performances of the cowpea (Vigna unguiculata Walp.) landraces in Southern Benin. International Research Journal of Agricultural Science and Soil Science, 3(4): 121-133.

Grenand F. 2002.Stratégies de nomination des plantes cultivées dans une société tupiguarani, les Wayãpi. Revue Amerindia, 26/27: 209-247.

Kombo GR, Dansi A, Loko LY, Orkwor GC, Vodouhe R, Assogba P, Magema JM. 2012. Diversity of cassava (Manihot esculenta Crantz) cultivars and its management in the department of Bouenza in the Republic of Congo. Genet. Resour. Crop. Evol., 59(8) : $1789-1803 . \quad$ DOI : 10.1007/s10722-012-9803-0

Kosh-Komba E, Akpavi S,Woegan YA, Atato A, Duval MF, Dourma M, Zinga I, Yandia P, Longue D, Semballa S. 2014. Diversité agromorphologique de Manihot Esculenta Crantz (Euphorbiaceae) cultivée dans trois zones agroclimatiques en République Centrafricaine (RCA). European Scientific Journal, 10(3): 365-380.

Labé V, Palm R. 1999. Méthodes et techniques. Statistique, empirique, formelle: quelle enquête pour la collection d'informations sur les exploitations agricoles? Cahiers Agricultures, 8(5): 397- 404.

Manu-Aduening JA, Lamboll RI, Dankyi AA, Gibson RW. 2005. Cassava diversity in Ghanaian farming systems. Euphytica,
144: $331-340$. DOI: $10.1007 / \mathrm{s} 10681-$ 005-8004-8

Manu-Aduening JA, Peprah B, Bolfrey-Arku G, Aubyn A. 2014. Promoting Farmer Participation in Client-oriented Breeding: Lessons from Participatory Breeding for Farmer-preferred Cassava Varieties in Ghana. Adv. J. Agric. Res., 2(2): 008-017.

Manusset S. 2006. Proposition pour une clé d'identification des variétés de manioc chez différents groupes culturels en Guyane française. Antropo, 11: 61-73.

McKey D, Emperaire L, Elias M, Pinton F, Robert $\mathrm{T}$, Desmoulière $\mathrm{S}$, Rival $\mathrm{L}$. 2001. Gestions locales et dynamiques régionales de la diversité variétale du manioc en Amazonie. Genet. Sel. Evol., 33: 465-490.

McKey D, Elias M, Pujol B, Duputié A, Delêtre M, Renard D. 2012. Maintien du potentiel adaptatif chez les plantes domestiquées à propagation clonale. Revue d'Ethnoécologie, URL: http://ethnoecologie.revues.org/741; DOI : 10.4000/ethnoecologie.741.

Missihoun AA, Agbangla C, AdoukonouSagbadja H, Ahanhanzo C, Vodouhe R. 2012. Gestion traditionnelle et statut des ressources génétiques du sorgho (Sorghum bicolor L. Moench) au NordOuest du Bénin. Int. J. Biol. Chem. Sci., 6(3): 1003-1018. DOI : http://dx.doi.org/10.4314/ijbcs.v6i3.8

Naïtormbaïdé M. 2012. Incidence des modes de gestion des fumures et des résidus de récolte sur la productivité des sols dans les savanes du Tchad. PhD Thèse, Université Polytechnique de BoboDioulasso, Burkina-Faso, p.192.

Nassar N, Ortiz R. 2010. Breeding cassava to feed the poor. Scientific American, 302 (5): 78-84.

N'Da HA, Akanvou L, Kouakou CK. 2013. Gestion locale de la diversité variétale du maïs (Zea mays L.) violet par les Tagouana au Centre-Nord de la Côte d'Ivoire. Int. J. Biol. Chem. Sci., 
7(5):2058-2068.

DOI: http://dx.doi.org/10.4314/ijbcs.v7i5.23

Obasi PC, Henri-Ukoha A, Anosike ON, Ibekwe UC. 2015. Net returns to cassava-based crop mixtures in Imo State, Nigeria. European Journal of Agriculture and Forestry Research, 3(1): 15-21.

Office National du Développement (ONDR) Rural. 2013. Rapport bilan d'activités de la campagne agricole 2013/14. Ministère de l'Agriculture et de l'Irrigation, p.43.

Pinton F, Emperaire L. 2001. Le manioc en Amazonie brésilienne : diversité variétale et marché. Genet. Sel. Evol., 33(1): 491-512.

Sardos J, McKey D, Duval MF, Malapa R, Noyer JL, Lebot V. 2008. Evolution of cassava (Manihot esculenta Crantz) after recent introduction into a South Pacific Island system: the contribution of sex to the diversification of a clonally propagated crop. Genome, $\mathbf{5 1}$ : 912-921. DOI: $10.1139 / \mathrm{G} 08-080$
Sawadogo N, Nebie B, Kiebre M, Kando PB, Nanema RK, Traore RE, Naoura G, Sawadogo M, Zongo JD. 2014. Caractérisation agromorphologique des sorghos à grains sucrés (Sorghum bicolor (L.) Moench) du Burkina Faso. Int. J. Biol. Chem. Sci., 8(5): 21832197.

DOI: http://dx.doi.org/10.4314/ijbcs.v8i5.22

Shannon CE.1948. A mathematical theory of communication. Bell System Technical Journal, 27: 379-423. DOI: 10.1002/j.1538-7305. 1948. Tb01338.x

Willemen L, Scheldeman X, Cabellos VS, Salazar SR, Guarino L. 2007. Spatial patterns of diversity and genetic erosion of traditional cassava (Manihot esculenta Crantz) in the Peruvian Amazon: An evaluation of socioeconomic and environmental indicators. Genet. Resour. Crop. Evol., 54(7): $\quad 1599-1612 . \quad$ DOI: 10.1007/s10722-006-9172-7 